\title{
Impacto de um programa de musicoterapia sobre o nível de estresse de profissionais de saúde
}

\author{
Impact of a music therapy program on the stress level of health professionals \\ Impacto de un programa de musicoterapia en el nivel de estrés de profesionales de la salud
}

\section{Gunnar Glauco De Cunto Taets', Claudio Joaquim Borba-Pinheiro", Nébia Maria Almeida de Figueiredo"II, Estélio Henrique Martin Dantas ${ }^{\text {III }}$}

' Universidade Federal do Estado do Rio de Janeiro, Escola de Enfermagem Alfredo Pinto, Programa de Pós-Graduação em Enfermagem e Biociências (Doutorando). Instituto D'Or de Pesquisa e Ensino, Hospital Barra D'Or. Rio de Janeiro-RJ, Brasil. "Universidade Federal do Estado do Rio de Janeiro, Escola de Enfermagem Alfredo Pinto, Programa de Pós-Graduação em Enfermagem e Biociências (Doutorando). Rio de Janeiro-RJ, Brasil.

II' Universidade Federal do Estado do Rio de Janeiro, Centro de Ciências Biológicas e da Saúde, Escola de Enfermagem Alfredo Pinto, Programa de Pós-Graduação em Enfermagem e Biociências. Rio de Janeiro-RJ, Brasil.

\section{Submissão: 30-07-2012 Aprovação: 22-05-2013}

\section{RESUMO}

Objetivou-se verificar os efeitos de um programa de musicoterapia sobre o nível de estresse de profissionais de saúde. Participaram do estudo 34 voluntários do sexo feminino com 33,3 \pm 8,5 anos de idade, de diferentes níveis de atuação profissional. Utilizouse o inventário de sintomas de Stress para adultos de Lipp (ISSL) para avaliar o nível de estresse dos participantes antes e após a musicoterapia. O programa foi composto por 12 sessões utilizando as técnicas musicoterápicas de Improvisação e Recriação Musical, realizado uma vez por semana com 50 minutos/sessão em um período de três meses. O teste de Wilcoxon para medidas repetidas foi utilizado para análise estatística. Observou-se uma diminuição estatística significativa $(\Delta=-60 \%$, $\mathrm{p}<0,001)$ no nível de estresse das profissionais estudadas após o programa de musicoterapia. Conclui-se com o presente estudo que o programa de musicoterapia foi efetivo para diminuir o nível de estresse de mulheres profissionais de saúde que atuam em um hospital privado no município do Rio de Janeiro-RJ.

Descritores: Esgotamento Profissional; Musicoterapia, Qualidade de Vida.

\section{ABSTRACT}

The study aimed to assess the effects of a music therapy program on the level of stress for female professionals working in a private hospital in Rio de Janeiro-RJ, Brazil. Thirty four female volunteers with $33.3 \pm 8.5$ years of age from different levels of professional participated in the study. We used the Lipp's inventory of symptoms of stress for adults (ISSL) to evaluate the level of stress of participants before and after music therapy. The program consisted of twelve sessions using the techniques of music therapy Improvisation and Musical Re-creation held once a week with 50 minutes / session in a period of three months. The Wilcoxon test for repeated measures was used for statistical analysis. The study showed a statistically significant decrease $(\Delta=-60 \%, p<0.001)$ in the level of stress professionals studied after the music therapy program. In conclusion, the present study that the music therapy program was effective in decrease the level of stress of women health professionals working in a private hospital in Rio de Janeiro-RJ, Brazil.

Key words: Burnout Professional; Music Therapy; Quality of Life.

\section{RESUMEN}

Objetivó-se evaluar los efectos de un programa de musicoterapia en el nivel de estrés para las mujeres profesionales que trabajan en un hospital privado en Rio de Janeiro-RJ, Brasil. Participaran del estudio 34 voluntarios de sexo femenino con 33,3 \pm 8,5 años de edad, de diferentes niveles de desempeño profesional. Se utilizó un inventario de síntomas de estrés para los adultos de Lipp (ISSL) para evaluar el nivel de estrés de los participantes antes y después de la terapia musical. El programa consistió en 12 sesiones que utilizan las técnicas de improvisación en musicoterapia y la re-creación musical a cabo una vez a la semana con 50 minutos por sesión en un plazo de tres meses. El test de Wilcoxon para medidas repetidas fue utilizado para el análisis estadístico. El estudio mostró una disminución estadísticamente significativa $(\Delta=-60 \%, p<0,001)$ en el nivel de estrés de los profesionales estudiados después de que el programa de terapia musical. En conclusión, el presente estudio demostró que el programa de musicoterapia fue efectivo en disminuir el nivel de estrés de los profesionales de la salud que trabajan en un hospital privado en Río de Janeiro-RJ, Brasil.

Palabras clave: Agotamiento Profesional; Musicoterapia; Calidad de Vida. 


\section{INTRODUÇÃO}

Atualmente a palavra estresse tem sido muito associada à sensação de desconforto, sendo cada vez maior o número de pessoas que se definem como estressadas ou relacionam a outros indivíduos na mesma situação ${ }^{(1)}$. Estressor é uma situação ou experiência que gera sentimentos de tensão, ansiedade, medo ou ameaça que pode ser de origem interna ou externa. A palavra stress começou a ser usada para expressar ação de força, pressão ou influência muito intensa sobre uma pessoa, causando nela uma deformação, assim como um peso suficiente pode envergar um pedaço de madeira(2).

O estresse, quando presente no indivíduo, pode desencadear uma série de doenças, dentre elas a depressiva ${ }^{(1)}$. Na área física, muitos tipos de doenças podem ocorrer, dependendo da herança genética, dentre elas: úlceras, hipertensão, crises de pânico, herpes e fibromialgia, e sem um tratamento especializado, existe o risco de ocorrência de problemas graves, como enfarte e acidente vascular encefálico ${ }^{(1,3)}$. Cabe ressaltar, que não é o estresse que causa essas doenças, mas propicia o desencadeamento de doenças para as quais há uma predisposição ou, ao reduzir a defesa imunológica, espaço para que doenças oportunistas apareçam ${ }^{(1)}$.

O quadro de stress relacionado ao ambiente ocupacional pode estar associado ao desenvolvimento da síndrome do burnout, na medida em que a sintomatologia característica deste quadro começa a aparecer em decorrência do desgaste causado pelo stress crônico ${ }^{(4-5)}$. Assim, o profissional estressado encontra-se debilitado, devido a investimentos de energia contra estressores, o que pode levá-lo a apresentar déficits em sua relação inter e intrapessoal e conseqüentemente em seu ambiente de trabalho ${ }^{(6-7)}$.

Estudos mostram que o nível de stress profissional tem sofrido um aumento nos últimos anos, principalmente em virtude dos avanços tecnológicos, das inovações com sobrecarga de tarefas no trabalho, da competição entre trabalhadores, da pressão relativa ao tempo de execução das tarefas de trabalho, dentre outros ${ }^{(5,8)}$. Segundo a Organização Mundial de Saúde (OMS), os locais de trabalho com um número considerável de estressores apresentam proporcionalmente um grande número de trabalhadores estressados, sendo $5 \%$ a $10 \% \mathrm{com}$ problemas graves ${ }^{(9)}$. Com isso, a proposta da atividade de musicoterapia para os profissionais que atuam na área saúde com o intuito de proporcionar um momento de descontração e principalmente que pudesse minimizar os efeitos do estresse do dia a dia pode justificar o presente estudo, pois estudos mostram que essa terapia pode minimizar os efeitos do estresse que acarretam como conseqüência, problemas na saúde física e mental ${ }^{(7,10)}$.

Utilizou-se neste estudo a definição de Musicoterapia da Federação Mundial de Musicoterapia ${ }^{(11)}$.

Musicoterapia é a utilização da música e/ou seus elementos (som, ritmo, melodia e harmonia) por um musicoterapeuta qualificado, com um cliente ou grupo, num processo para facilitar e promover a comunicação, relação, aprendizagem, mobilização, expressão, organização e outros objetivos terapêuticos relevantes, no sentido de alcançar necessidades físicas, emocionais, mentais, sociais e cognitivas. A Musicoterapia objetiva desenvolver potenciais e/ ou restabelecer funções do indivíduo para que ele/ela possa alcançar uma melhor integração intra e/ou interpessoal e, conseqüentemente, uma melhor qualidade de vida, pela prevenção, reabilitação ou tratamento.

Com isso, o objetivo deste estudo foi verificar os efeitos de um programa de musicoterapia sobre o nível de estress, de profissionais do sexo feminino que atuam em um hospital privado no Rio de Janeiro.

\section{MATERIAIS E MÉTODOS}

\section{Participantes}

O estudo contou com 34 voluntários do sexo feminino de diferentes áreas de atuação profissional de um hospital privado na cidade do Rio de Janeiro. O estudo teve caracteristicas de intervenção terapeutica com pré e pós testes em um único grupo.

\section{Critérios de inclusão e exclusão}

Os voluntários incluídos no estudo foram os funcionários do sexo feminino, com idade igual ou superior a 18 anos e com atuação efetiva no hospital privado estudado. De outra forma, foram excluídos os funcionários que fizessem uso de medicação estimulante do sistema nervoso, antidepressivos ou que estivesse com acompanhamento psicoterápico com outro profissional, além daqueles com qualquer tipo de licença de trabalho. Dessa forma, 34 voluntários de diferentes áreas de atuação profissional foram selecionados, que são mostrados na Figura 1.

Figura 1 - Distribuição de frequência dos profissionais, segundo categoria.

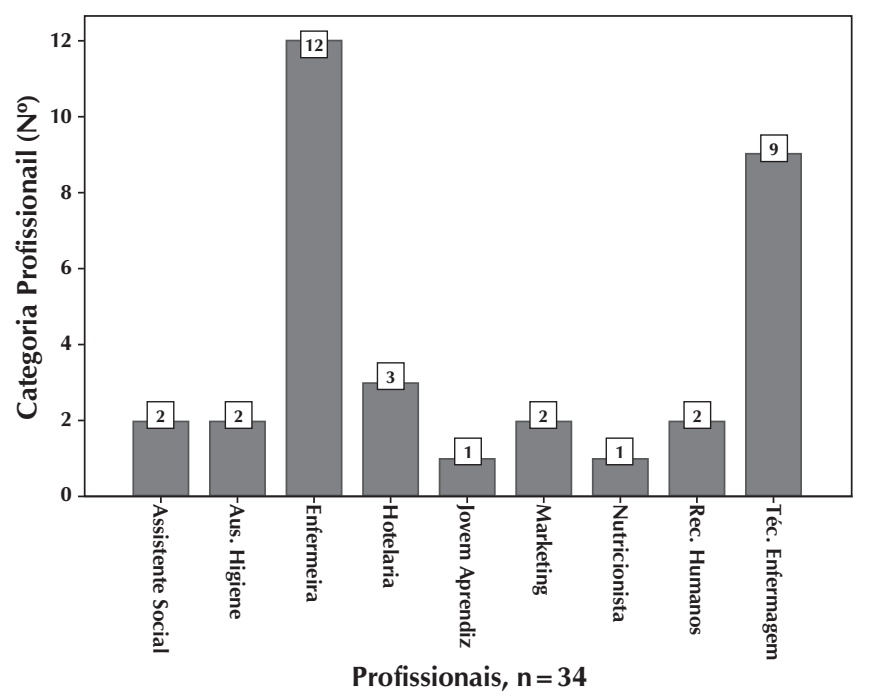

O projeto de pesquisa foi aprovado pelo Comitê Científico do Instituto D'Or de Pesquisa e Ensino e pelo Comitê de Ética em Pesquisa do Hospital Copa D'Or com protocolo de $n^{\circ}$ 
233/11. Os sujeitos de pesquisa que concordaram em participar do estudo assinaram o Termo de Consentimento Livre e Esclarecido em cumprimento a Resolução 196/96 do ConseIho Nacional de Saúde ${ }^{(12)}$.

\section{Protocolos para avaliação}

Utilizou-se para diagnóstico de estresse o Inventário de Sintomas de Stress para Adultos de Lipp (ISSL) ${ }^{(13)}$ para mostrar em que fase de estresse os sujeitos da pesquisa se encontravam. O ISSL foi aplicado e analisado por um profissional de psicologia.

Para avaliação do Estresse foi criado um questionário semi-estruturado que foi aplicado antes e após a terapia com musicoterapia pelo pesquisador com as seguintes perguntas:

1. Você se acha estressado?

2. Você considera o seu trabalho estressante?

As respostas, em escala Likert ${ }^{(14-15)}$, poderiam ser: (1) nenhum pouco / nada, (2) muito pouco, (3) pouco, (4) moderadamente e (5) muito.

\section{Procedimento de intervenção}

As sessões de musicoterapia foram chamadas de Encontros Musicais e ocorreram no auditório do Hospital duas vezes por mês, quinzenalmente, com duração de uma hora/sessão, realizadas no período de Dezembro de 2011 a Maio de 2012 com um total de 12 sessões.

As técnicas musicoterápicas utilizadas foram a "Improvisação e a Re-criação Musical", nas quais os sujeitos de pesquisa puderam se expressar utilizando a música através da atividade de Canto Coral. O repertório musical utilizado nas sessões foi composto por diversos gêneros de música popular brasileira e também de músicas internacionais escolhidas pelos sujeitos da pesquisa acompanhadas por um teclado tocado pelo pesquisador.

A teoria que fundamenta este estudo é a teoria humanista que, segundo Maslow, afirma que o ser humano, independentemente das circunstâncias que o envolve, possui a capacidade inata de assumir o controle da sua vida e de promover o seu próprio desenvolvimento por meio das capacidades humanas de escolha, criatividade e autorrealização. Maslow desenvolveu a teoria da motivação centrada no conceito da autorrealização que transmite o "desenvolvimento máximo dos potenciais de cada ser humano"(16).

\section{Análise Estatística}

Utilizou-se o programa IBM ${ }^{\circledast}$ SPSS $^{\oplus} 20.0$ for Windows para análise dos dados. Inicialmente, foi realizada uma descrição da amostra com média, mediana, desvio padrão, número máximo e mínimo dos dados de entrada da amostra estudada. A escala ordinal do instrumento de avaliação da veriável dependente, seguida da verificação da normalidade pelo teste de Shapiro Wilk, determinou a escolha do teste não paramétrico de Wilcoxon para medidas repetidas aceitando o nível de significância de $p<0,01$. Para calcular o delta percentual utilizou-se a fórmula $[\Delta=$ (Pósteste - teste) $* 100 /$ Teste $]$.

\section{RESULTADOS}

A Tabela 1 apresenta uma estatística descritiva dos dados de entrada, com valores de média, mediana, desvio padrão, número máximo e mínimo das variáveis do grupo estudado. A mediana mostra na Tabela 1 que o nível de estresse do grupo classifica-se em moderado de acordo com a escala Likert proposta.

Tabela 1 - Descrição da amostra estudada.

\begin{tabular}{|c|c|c|c|c|c|}
\hline \multicolumn{6}{|c|}{ Grupo Musicoterapia, $n=34$} \\
\hline Variáveis & Média & Md & DP & $N^{\circ}{ }_{\text {Máx }}$ & $\mathrm{N}^{\circ}$ \\
\hline Idade (anos) & 33,3 & 32,5 & 8,5 & 54,0 & 19,0 \\
\hline Nível de Estresse (Pontos) & 3,5 & 4,0 & 1,1 & 5,0 & 1,0 \\
\hline $\begin{array}{l}D P=\text { Desvio Padrão; } \\
M d=\text { Mediana; } \\
N^{\circ}=\text { Número Máxim } \\
N^{\circ}{ }_{\text {Min }}^{\text {Máx }}=\text { Número Mínimo }\end{array}$ & & & & & \\
\hline
\end{tabular}

O Inventário de Sintomas de Stress mostrou uma alta incidência de estresse nos profissionais participantes do estudo, dos quais 32 (94\%) foram classificados como estressados e somente 02 (6\%) não estressados.

A Figura 2 apresenta os resultados referentes a aplicação da musicoterapia para avaliar o estress de profissionais liberais do sexo feminino, mostrando que houve uma diminuição estatística significativa de $\Delta=-60 \%$ no nível de estress após a terapia.

Figura 2 - Análise dos efeitos da Musicoterapia sobre os níveis de estresse. $O$ símbolo $(*)$ indica o valor de $p<0,01$.

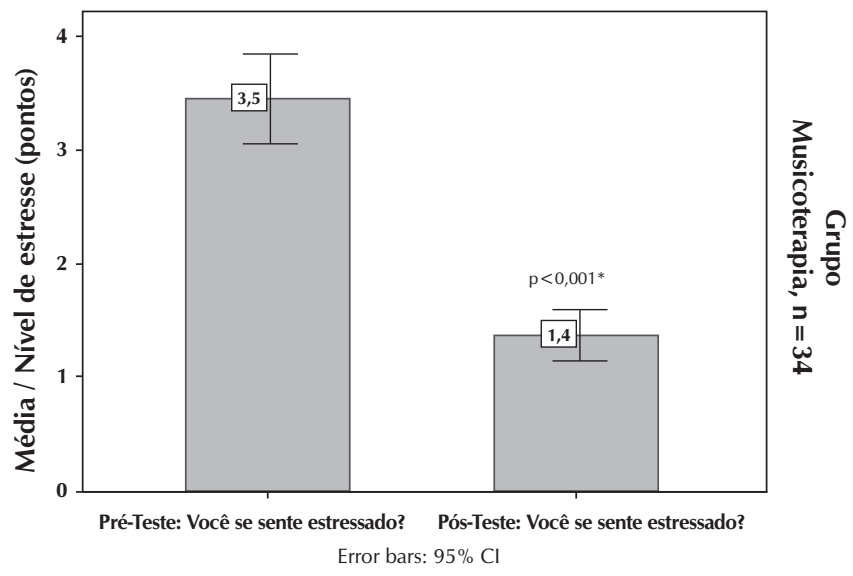

\section{DISCUSSÃO}

De acordo com as considerações de Hatem ${ }^{(17)}$ a ciência começa a investigar o efeito da música sobre as funções fisiológicas dos seres humanos, dentre elas a frequência cardíaca, respiratória e os efeitos vibratórios gerais no final do século XVIII, e com isso, iniciaram-se estudos de investigação sobre a relação da música com as respostas fisiológicas e psicológicas do organismo humano. Gatti e Silva(18) afirmam que já no século XIX, a música vinha sendo utilizada pelas instituições 
psiquiátricas, onde já era possível observar que a audição de melodias suaves era capaz de acalmar pacientes agitados, o que é corroborado por Nunes-Silva et al. ${ }^{(19)}$ pois estes também afirmam que as músicas consideradas sedativas, com andamento lento e poucas variações rítmicas, podem reduzir o estresse e favorecer o relaxamento, sinalizando de forma positiva para sua utilização como um instrumento facilitador do processo terapêutico.

Em estudo realizado com futuros profissionais, estudantes universitários, que estavam em tratamento para a depressão e que se encontravam sob um crescente estresse por diferentes motivos, dentre os citados: o conflito com o companheiro de quarto, problemas acadêmicos, preocupações financeiras e de carreira, além da pressão da família, foi constatado que os métodos não-médicos de enfrentamento, entre eles, escutar música foi frequentemente citado pelos voluntários como mais eficaz do que a terapia medicamentosa tradicional ${ }^{(20)}$.

Além do estresse de estudantes universitários, o estresse no trabalho também tem sido alvo de estudos na comunidade científica devido aos efeitos negativos para a saúde física e mental dos trabalhadores ${ }^{(7,11,19)}$.

No presente estudo, constatou-se que 32 (94\%) dos profissionais participantes encontravam-se estressados e $02(6 \%)$ não estressados. O fato de mais de $90 \%$ dos profissionais que participaram do estudo se encontrar estressados gera preocupação quanto à sua atuação profissional e qualidade de vida Dessa forma, os estudos com musicoterapia têm contribuído para a minimização dos efeitos estressantes do trabalho e conseqüentemente para a melhora da qualidade de vida ${ }^{(7,11,17-19)}$.

Todos os profissionais estudados no presente estudo encontravam na fase 2 do estresse, ou seja, resistência, cujos sintomas podem interferir sobremaneira nas diferentes esferas de suas vidas pessoal e profissional, uma vez que a característica principal desta fase é o grande requerimento de energia adaptativa do organismo em prol do restabelecimento da homeostase interna perdida na fase anterior (alerta) ${ }^{(1)}$.

No que se refere à relação entre stress e profissão, na medida em que se encontrou 32 profissionais estressados (94\%) sendo que todos desenvolviam atividades assistenciais, supõe-se, com tal resultado, que a prática da assistência leva o profissional ao contato direto com o sofrimento, o tornando mais vulnerável ao stress. Esses dados condizem com a literatu$\mathrm{ra}^{(5,17-19)}$, que chama a atenção para o desenvolvimento da síndrome de burnout em profissionais que prestam assistência.

O estresse pode desencadear uma série de doenças, se, nada for feito para minimizar a tensão, podendo levar dentre outras doenças, a depressão ${ }^{(6,11,19)}$ reforçam afirmando que, sem um tratamento especializado que considere as características pessoais, existe além da depressão, um risco aumentado da ocorrência de problemas mais graves, como enfarte e acidente vascular encefálico.

Entretanto, não é o estresse que causa essas doenças, mas ele pode ser o agente de desencadeamento para as quais a pessoa tenha predisposição ou, ao reduzir a defesa imunológica, abre espaço para que doenças oportunistas apareçam ${ }^{(7)}$.

Em um estudo que verificou o efeito de uma sessão de terapia musical sobre mulheres com fibromialgia, lesão por esforço repetitivo e doenças osteoarticulares, constatou-se uma redução estatística $(p<0,001)$ na intensidade da dor após uma sessão de audição musical ${ }^{(3)}$. Em outro estudo(21), que avaliou a fadiga compassiva e a formação de grupos para o trabalho entre enfermeiros, assistentes sociais e outros profissionais de um hospital psiquiátrico, separados em dois grupos com dois distintos programas de musicoterapia, no qual, o primeiro grupo usou uma abordagem ecológica que privilegia os temas livres, com improviso de canto e instrumentos musicais, já outro grupo, usou uma abordagem didática, com intervenções planejadas incluindo meditação guiada com música ao vivo, análises das letras das músicas e movimentação.

Os resultados deste estudo indicaram uma melhora significativa $(p<0,05)$ para a construção da equipe de trabalho em ambos os grupos, mas não houve diferenças significativas em relação à fadiga compassiva.

De acordo com as considerações de Guazina e Tottoni ${ }^{(22)}$ as abordagens direcionadas exclusivamente a população geral não atinge a coletividade no ambiente de trabalho, em especial no ambiente hospitalar, de modo a interferir na melhoria organizacional deste ambiente, uma vez que, o atendimento com musicoterapia em técnicos de enfermagem de uma Unidade de Terapia Intensiva infantil de um hospital público da cidade de Porto Alegre, refaz, "relaxa", retoma melhores condições físicas e psíquicas neste profissional, embora, ainda exista resistência na implantação deste tipo de terapia nos ambientes de hospitais públicos.

No presente estudo também foi mostrado benefícios através da metodologia com musicoterapia que diminuiu em $60 \%$ a percepção de estresse nos participantes com diferença estatística significativa $(p<0,01)$ apresentado na Figura 2 , demonstrando que a referida terapia pode ser um instrumento de auxílio para minimização do estresse nos profissionais que atuam em hospitais. Outros estudos corroboram com os achados apresentados na presente pesquisa, como o que foi realizado com musicoterapia e após 16 sessões apresentou dentre outras variáveis, um aumento no nível de satisfação pessoal de $44,5 \%$ e uma diminuição no nível de estresse de $66,7 \%$ nos participantes ${ }^{(23)}$.

Outro estudo, que realizou uma sessão de alongamentos com 54 voluntários separados em dois grupos, quais sejam: grupo de alongamentos com músicas preferidas e grupo de alongamentos músicas "New Age", sendo utilizado o ISSL para o nível de estresse, verificou-se neste estudo que ambos os grupos provocaram uma redução significativa $(p<0,05)$ no nível de estresse, mostrando também, que a preferência musical não foi determinante para influenciar a redução do estresse ${ }^{(24)}$.

Em estudo realizado com 49 profissionais do pronto-socorro adulto de um hospital privado utilizando a musicoterapia com repertório erudito ${ }^{(18)}$, observou-se que, com a presença da música, $78 \%$ dos profissionais percebeu alteração no ambiente, $41 \%$ acreditou que a música alterou seu desempenho pessoal, de forma positiva para $85 \%$ e negativa para $15 \%$. Em relação ao repertório musical, $61 \%$ dos indivíduos gostaram da seleção, 96\% acredita que se deve continuar com a música ambiente com sugestão de outros gêneros musicais por $76 \%$ dos entrevistados. 
Em um estudo de revisão sistemática ${ }^{(25)}$ que avaliou a eficácia da musicoterapia em comparação com o tratamento padrão para pessoas com depressão e comparar os efeitos da musicoterapia nestas pessoas em relação a outros tratamentos psicológicos ou farmacológicos utilizando como critério a seleção de estudos clínicos randomizados. Neste estudo, cinco trabalhos preencheram os critérios de elegibilidade nos quais quatro destes foram considerados efetivos para o controle da depressão em relação aos tratamentos convencionais.

Portanto, os estudos supracitados reforçam e corroboram os achados da presente pesquisa, que mostrou na musicoterapia uma alternativa para minimização do estresse de profissionais ligados à área da saúde. O presente estudo, dentro das limitações da amostra, demonstrou que a musicoterapia foi uma importante ferramenta para minimizar o estresse do dia a dia dos profissionais de saúde visto que houve uma diminuição estatística $(p<0,01)$ na percepção de estresse nos sujeitos da pesquisa depois da sessão de musicoterapia.

Contudo, os dados obtidos nesse estudo sugerem a necessidade de novas pesquisas sobre o tema com um maior universo de abrangência com um grupo de controle. A temática do estresse de colaboradores deve ser vista com um olhar preventivo e uma vez identificado, deve se tentar promover ações, como a utilização da musicoterapia, por exemplo, para que não chegue a níveis de exaustão acarretando em queda da qualidade no desempenho de suas funções e até mesmo em afastamento do trabalho.

\section{CONCLUSÃO}

Conclui-se com o presente estudo que o programa de musicoterapia foi efetivo para diminuir o nível de estresse de mulheres profissionais de saúde que atuam em um hospital privado no Rio de Janeiro-RJ em $\Delta=-60 \%$.

\section{REFERÊNCIAS}

1. Coser O. Depressão: clínica, ética e crítica. Rio de Janeiro: Fiocruz, 2003.

2. Lipp MEN. Stress e suas implicações. Rev Psicol 2009;3(4):5-19.

3. Leão ER, Silva MJP. Música e dor crônica musculoesquelética: o potencial evocativo de imagens mentais. Rev Latino-Am Enferm 2004;12(2):235-41.

4. Román Hernández J. Estrés y Burnout en Profesionales de la Salud de los Niveles Primario Y Secundario de Atención. Rev Cuba Salud Pública 2003;29(2):103-110.

5. Malagris LEN. Burnout: o profissional em chamas. In: Sobrinho FPN, Nassaralla I, editores. Pedagogia Institucional: fatores humanos nas organizações. Rio de Janeiro: ZIT; 2004. p. 196-213.

6. Salomé GM, Martins MFMS, Espósito VHC. Sentimentos vivenciados pelos profissionais de enfermagem que atuam em unidade de emergência. Rev Bras Enferm 2009;62(6):856-62.

7. Taets GGC, Barcellos LRM. Música no cotidiano de cuidar: um recurso terapêutico para enfermagem. Rev Pesqui Cuid Fundamental (online) 2010;2(3):1009-1016.

8. Pafaro RC. Estudo do estresse do enfermeiro com dupla jornada de trabalho em um hospital de oncologia pediátrica de Campinas. Campinas. Dissertação [Mestrado em Enfermagem] - Universidade Estadual de Campinas; 2002.

9. Mendes R. Patologia do Trabalho. Rio de Janeiro: Ateneu, 1995.

10. Zorzi Gatti MF, Ribeiro Leão E, Paes Silva MJ, Giesbrecht PA. Comparación entre los niveles de ansiedad y stress presentados y percibidos por el colectivo de enfermería. Rev Enfermería Global 2004;3(2):1-12.

11. Musicoterapia Brasil [homepage na internet]. Federação Mundial de Musicoterapia [acesso em: 23 maio 2013. Disponível em<http://www.musicoterapiabrasil.org/
Novo/ portal/index.php?option $=$ com_content\&task $=$ vie w\&id $=13 \&$ Itemid $=30>$.

12. Ministério da Saúde (Brasil). Resolução $n^{\circ}$. 196, de 10 de outubro de 1996. Normas para a Realização de Pesquisa em Seres Humanos. Diário Oficial da União 1996;Seção 1.

13. Lipp MEN. Inventário de Sintomas de stress para adultos de LIPP (ISSL). São Paulo: Casa do Psicólogo; 2000.

14. Likert RA. Technique for the Measurement of Attitudes. Archives of Psychology 1932;22(140):1-55.

15. Cunha LMA. Modelos Rasch e Escalas de Likert e Thurstone na medição de atitudes. Lisboa. Dissertação [Mestrado em Estatística] - Faculdade de Ciências da Universidade de Liboa; 2007.

16. Carmo ALD. Formação humanista frente à crise de valores gerada pela globalização [periódico na internet]. 2012 [acesso em: 21 mar 2012. Disponível em http://www. conexaeventos.com.br/detalhe_artigo.asp?id=35. 2009.

17. Hatem TP, Lira PIC, Matto SSS. Efeito terapêutico da música em crianças em pós-operatório de cirurgia cardíaca. J Pediatr 2006;82:186-192.

18. Nunes-Silva M, Moreira LC, Moraes GRJ, Rosa GT, Marra CAS. A música para indução de relaxamento na Terapia de Integração Pessoal pela Abordagem Direta do Inconsciente - ADI/TIP. Contextos Clínicos 2012;5(2)88-99.

19. Gatti MFZ, Silva MJP. Música ambiente em serviço de emergência: percepção dos profissionais. Rev Latino-Am Enferm 2007;15(3):377-383.

20. Aselton P. Sources of stress and coping in American college students who have been diagnosed withdepression. J Child Adolesc Psychiatr Nurs 2012;25(3):119-23.

21. Hilliard RE. The effect of music therapy sessions on compassion fatigue and team building of professional hospice caregivers. The Arts in Psychotherapy 
Taets GGC, et al.

2006,33(5):395-401.

22. Guazina L, Tittoni J. Musicoterapia institucional na saúde do trabalhador: conexões, interfaces e produções. Psicol Soc 2009;21(1):108-117.

23. Marconato C, Munhoz EC, Menim MM, Albach MT. Aplicação da Musicoterapia Receptiva na Clínica Médica e
Cardiológica. Arq Bras Cardiol 2001;77(2):138-9.

24. Valim PC, Bergamaschi EC, Volp CM, Deutsch S. Redução de Estresse pelo Alongamento: a Preferência Musical Pode Influenciar? Motriz 2002;8(3).

25. Maratos A, Gold C, Wang X, Crawford M. Music therapy for depression. Cochrane Database Syst Rev 2008;1. 